\title{
Importance of Digital Literacy in the Process of Confronting the Stress During COVID-19 Pandemic
}

\author{
Daša BOSANAC ${ }^{\mathrm{a}, 1}$ and Ljerka LUIC ${ }^{\mathrm{a}}$ \\ ${ }^{\text {a }}$ University North, Croatia
}

\begin{abstract}
COVID-19 pandemic is a global problem that raised the perception of stress to a completely new level, unseen so far in peace conditions. To reduce the level of stress and deal with its accumulated consequences, people enforce different strategies and coping mechanisms. In the vast amount of information available, imposed by the media, and often filled with falsehoods and catastrophizing related topics, it can be very challenging to distinguish the truth from falsehoods. The existence of an adequate level of digital literacy and e-Health literacy can help society to deal with that problem. Digital and e-Health literacy enables society to search for information efficiently, while remaining critical and open-minded, and use the newly adopted knowledge to improve our mental health. The aim of the paper was to present the frequency of news searches and trusting the COVID-19 related information in the group of medical professionals in Croatia. The survey was conducted using an online questionnaire, containing psychological questionnaires and relevant questions about everyday functioning. Based on the results that show a significant correlation between the frequency of information search and the lack of trust in the search results, we discuss the importance of digital and e-Health literacy, primarily within medical professionals.
\end{abstract}

Keywords. Digital literacy, media literacy, e-Health literacy, coping mechanism

\section{Introduction}

COVID-19 pandemic is a new global problem that engulfed the entire world and affected all aspects of society. The threat of infection with the SARS-CoV-2 virus is causing fear and uncertainty throughout the population and it has a major influence on public behavior. Enforced preventive measures are a real threat to the mental health of individuals and result in an increased level of stress.

Stress is an evolutionary answer to the threat that we all react in different ways, with emotional, physical, mental, and behavioral reactions [1]. When under stress, people have a natural need to reduce its intensity. To achieve that, they use different strategies and coping mechanisms. It is possible to experience denial, loneliness, but also increased risk of aggressive and suicidal behavior [2]. This is something we all witness on a daily basis. Violent demonstrations due to limitations of personal freedoms, and directly increased stress levels are currently emerging in highly develop, technocratic western countries like France and Holland.

${ }^{1}$ Corresponding Author, Daša Bosanac, University North, Trg dr.Zarka Dolinara 1, 48000 Koprivnica, Croatia; E-mail: dabosanac@unin.hr. 
Buljan-Flander [1] published a complete list of typical stressors related to COVID19, two of which are related to information: Lack of clear information and Media information exposure. Constant everyday bombardment with information from the media and the means of their relay with the tendency of over exaggerating and even catastrophizing are having a major influence on the quality of life. Availability of information has never been greater. At the same time, the quality of information is significantly decreased, and fake news is not leaving the front lines. Various conspiracy theorists are gaining popularity, and are using all means and channels at their disposal to distribute the content they are fabricating. Social networks are being the most popular and effective way. This phenomenon is defined as decentralization of information, where users apart from the consumer status also participate in the creation of new information and content [3]. Technical and intellectual skills required to critically evaluate and absorb the information distributed through the mass media are becoming more and more important. The ability to approach the information filtering biased and fake news is considered a key factor while evolving into the new generation of smart users [4]. These abilities are defined as digital and media literacy. Digital literacy is defined as the ability of the individual to find, evaluate, comprehend and compose clear information on various digital media and platforms. It requires "a large variety of complex skills such as cognitive, motor, sociological and emotional skills that users need to master in order to use digital environments effectively" [5]. Media literacy is generally defined as the ability to access the media, read, understand, and critically evaluate available information [6].

The use of digital technologies to support, deliver and enhance mental health services, and therefore generally improve the mental health and well-being of individuals is known as e-Mental Health (e-MH) [7]. e-MH services and interventions "can be categorized in terms of their purposes, such as providing information, screening, assessment, monitoring, intervention, and peer support" [8]. "Interventions can include self-management tools, communication with service providers, counseling, and various forms of psychosocial therapies.

In addition to the term e-Health, e-Health literacy is defined as "the ability to seek, find, understand and appraise health information from electronic sources and apply knowledge gained to addressing or solving a health problem."[9,10]. Having the compound complex skills of e-Health literacy allows consumers to achieve positive outcomes from using the Internet for health purposes. e-Health "literacy has the potential to both protect consumers from harm and empower them to fully participate in informed health-related decision making"[10]. People with well-developed e-Health literacy are also more aware of the risk of coming across unreliable information on the Internet [11]. While being constantly bombarded with fake news and biased reports related to COVID-19, high e-Health literacy can be seen as a good predictor of adequate coping mechanisms.

In addition to the psychological problems related to the pandemic conditions, we were interested in how medical professionals, as those who are involved in patients' treatment process and whom patients should trust most, react to the newly developed situation. We focused on health workers as they were additionally exposed to stress due to their role during the pandemic. We were interested how often they search for news related to the pandemic and how much they trust the information itself. We hypothesized that they tend to search the Internet more often during the pandemic, but also that they are more critical of the news and trust them less. 


\section{Methods}

We have conducted research related to definitions of medical professionals' mental health in a public health institution in Croatia. The goal of the research was to test satisfaction of life, perceived levels of stress, anxiety, and depression related to the pandemic. An additional goal was to test the coping strategies and the mean to adapt to changed living conditions, caused by the pandemic [12].

The survey was conducted using the Google Form online questionnaire, from April till June 2020. Consequently, 147 respondents $(\mathrm{N}=147), 16$ male and 131 female, age average 39 years, participated in the survey. The questionnaire consisted of large number of psychological symptom and personality scales, coping mechanism scales, and general questions about functioning during the lockdown, which was from March till May $2020[12,13]$.

For the purpose of this paper we did an explanatory study and focus on the responses of those variables that examined the increased frequency of information search on the internet, the increased frequency of information retrieval about Covid-19, and trust in information gained. To access relationship between those variables, we applied Spearmans coefficient of correlation.

\section{Results}

Respondents reported a generally high level of life satisfaction, usage of good coping mechanisms, and anticipation of positive well being. They also reported low fear of getting sick, low level of propensity to disrupt partnership and family relationships [12].

According to the use of media, respondents reported that in the period of lockdown, from March till May 2020, they manifested more news reading and more information search about COVID-19. They also reported about lack of trust in information (Figure 1).

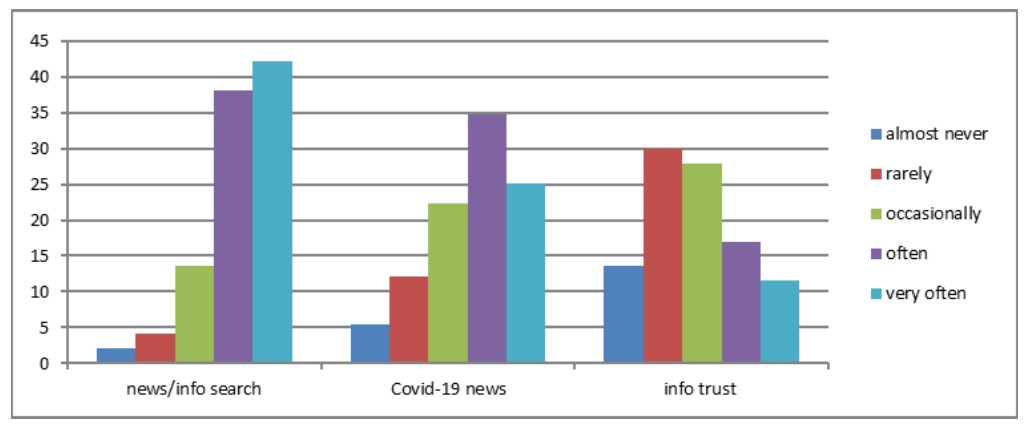

Figure 1. Percentage of reading and searching information and belief in gained information.

Statistically significant correlation was found between more frequent news following in general and lack of trust in information, in a direction that those who were following the news more frequently were expressing doubt and lack of trust in the gained information $(\mathrm{r}=0,324 ; \mathrm{p}<0,001)$. Statistically significant correlation was also found between searching information about COVID-19 and lack of trust in it $(r=0,340$; 
$\mathrm{p}<0,001)$. Participants who wanted to know more about COVID-19, and for that reason search for the information on the Internet, reported about lack of trust in information.

\section{Discussion}

Our results, as expected, showed that more than $70 \%$ of medical health professionals frequently searched for information and followed news on the internet during the pandemic. Also, over $50 \%$ of respondents expressed in lack of trust in information on the internet. That indicates a well-developed Digital and e-Health literacy among the medical professionals in a public health institution in Croatia. This may be explained by the participants who were medical professionals and thus confident in their knowledge about disease and search skills. Their work and profession enable them to understand medical and scientific publications about the virus and perceive information on the internet with a sufficient level of criticism.

Our participants also reported about the high level of life satisfaction, usage of good coping mechanisms, and anticipation of positive well-being [12], all of which are indicators of good mental health. High e-Health literacy can also be considered as the guardian of our mental health [10]. According to that, developed e-Health literacy in our sample could be one of the reasons for a high level of life satisfaction. New studies are required to obtain information about the connection between lack of e-Health literacy and stress and burn-out. Here we need to point out how convenient our sample is, not only by profession but also by gender and digital literacy. It is likely that only those respondents, who use the internet and those who are familiar with completing online surveys, completed the questionnaire. That limitation of the study points out the question of what data would be obtained from the sample of higher gender and age dispersion.

The results can also be explained by the higher than expected exposure to false information and subsequently, lack of trust towards information related to COVID-19. That emerges the question of how influential the media really are, concerning the lack of trust that subjects are expressing towards information received.

Persons prone to thinking are usually better at recognizing fake news and false information. If we are not directly involved in the subject, we are inclined to accept the offered information almost automatically, and with little or no resistance. If we devout just a little bit of our time to a certain subject, unbiased and as critical as possible, we will become much better at recognizing fake news [14]. Our cognitive system is usually overstretched, with the huge influx of incoming information from one side, to the need for quick actions on the other side. If we free up some of our time, by disabling the practice of fast scrolling through the news, and give our self the chance to think about what we just read critically, we will be much less prone to believing fake news [14 ].

In addition to exposure to falsehoods, the impact of social networks, especially among the young, further complicates the progress of digital literacy. Numerous Facebook groups and Reddit forums offer platforms for discussion on just about any topic, without checking information. Deep fake videos are reaching the level of being indistinguishable from the real ones. The first step of fighting against this type of propaganda is to ask yourself the question. "Is this really possible? Is the person in 
question likely to behave in the way as described in the video?" The next step would be looking for confirmation and verification of the news from a reliable source.

\section{Conclusions}

Medical health professionals in Croatia show high e-Health literacy and criticism toward information gained on the internet. Well-developed e-Health literacy from the psychological perspective can be considered as the guardian of our mental health and consequently good confrontation strategy. New research is required with an emphasis on the relationship between e-Health literacy, stress, and general burnout.

\section{References}

[1] BuljanFlander G, Bogdan A. Connected: Telephone and Online Counseling in Coping with the COVID-19 Pandemic and its Consequences. Croatian Chamber of Psychology, the City of Zagreb, City Office for Health and Zagreb Child and Youth Protection Center, 2020.

[2] Li W, Yang Y, Liu ZH, Zhao YJ, Zhang Q, Zhang L, Cheung T, Xiang YT. Progression of Mental Health Services during the COVID-19 Outbreak in China. International Journal of Biological Sciences. 2020;16(10):1732.

[3] Bruns A. Blogs, Wikipedia, Second Life, and beyond: from production to produsage. New York, New York: Peter Lang, Publishing; 2008.

[4] Buckingham D. Media education and the end of the critical consumer. Harvard Educational Review. 2003;73(3):309-328.

[5] Eshet Y. Digital literacy: A revised model. Issues in Informing Science and Information Technology. 2012;9:267-76. DOI: 10.28945/1621.

[6] Škoda J, LuićL,Brlek E. Informational aspects of digital literacy. EDULEARN20 Proceedings. 2020. doi:10.21125/edulearn.2020.1064.

[7] WPA Position statement on e-Mental Health, 2018. Available at: https://www.wpanet.org/positionstatements, Accessed 21 January 2021.

[8] Lal S, Adair CE. E-mental health: a rapid review of the literature. Psychiatric Services. 2014 Jan 1;65(1):24-32. doi: 10.1176/appi.ps.201300009.

[9] Norman C, Skinner H. eHEALS:the eHealth Literacy Scale. Journal of Medical Internet Research. 2006;8(4):e27. doi:10.2196/jmir.8.4.e27.

[10] Hayat T, BraininE,Neter E. With Some Help From My Network: Supplementing eHealth Literacy With Social Ties. Journal of Medical Internet Research. 2017;19(3):e98. doi:10.2196/jmir.6472. PMC 5391437. PMID 28360024.

[11] Neter E, Brainin E. eHealth literacy: extending the digital divide to the realm of health information. Journal of Medical Internet Research. 2012;14(1):e19. doi:10.2196/jmir.1619. PMC 3374546. PMID 22357448

[12] Bosanac D, Dadic-Hero E, Hero L. (in press). Psychological Health of medical staff in the Corona virus epidemic.

[13] Cubela Adoric V, Buric I, Macuka I. 10 volumes of the collection of Psychological Scales and Questionnaires. In book: Zbirka psihologijskih skala i upitnika. University of Zadar; 2020.

[14] Pennycook G, Rand DG. Fighting misinformation on social media using crowdsourced judgments of news source quality.PNAS, Proceedings of the National Academy of Sciences of the United States of America. 2019; Vol.116 (7):2521-2526. doi:10.1073/pnas.1806781116. 